Amir Shams, ${ }^{* 1}$ Ahmad Ali Akbari Kamrani, ${ }^{2}$

Parvaneh Shamsipour Dehkordi, ${ }^{1}$ Robabeh Mohajeri ${ }^{3}$

\title{
MODERATE-INTENSITY AEROBIC EXERCISE IMPROVES SLEEP QUALITY IN MEN OLDER ADULTS
}

\begin{abstract}
The purpose of present research was to investigate the effect of low and moderate intensity aerobic exercises on sleep quality in older adults. The statistical sample included 45 volunteer elderly men with age range of 60-70 years old that divided randomly in two experimental groups (aerobic exercise with low and moderate intensity) and one control group. The maximum heart rate (MaxHR) of subjects was obtained by subtracting one's age from 220. Furthermore, based on aerobic exercise type (40-50\% MaxHR for low intensity group and 60-70\% MaxHR for moderate intensity group) the target MaxHR was calculated for each subject. The exercise protocol consisted of 8 weeks aerobic exercises ( 2 sessions in per-week) based on Rockport one-mile walking/running test and the control group continued their daily activities. All subjects in per-test and post-test stages were completed the Petersburg Sleep Quality Index (PSQI). Results in post-test stage showed that there were significant differences between control and experimental groups in sleep quality and its components $(\mathrm{P}<0.05)$. Also, the Tukey Post Hoc showed that the moderate intensity group scores in sleep quality and its components were better than other groups $(\mathrm{P}<0.05)$. Finally, the low intensity group scores were better than control group $(\mathrm{P}<0.05)$. Generally, the present research showed that the aerobic exercises with moderate intensity have a positive and significant effect on sleep quality and its components. Thus, based
\end{abstract}

\footnotetext{
* Corresponding author: Amir Shams Email: amirshams85@gmail.com

Tel: 989196823597 , Fax: 982129902937

Mailing address: Physical Education and Sport Science Faculty, Shahid Beheshti University, Daneshjoo Blv., Evin, Tahran, Iran.

${ }^{1}$ Department of Physical Education and Sport Sciences, Shahid Beheshti University, Tehran, Iran.

${ }^{2}$ Social-Psychological Research Center on Aging, University of Social Welfare \& Rehabilitation Sciences, Tehran, Iran.

${ }^{3}$ Islamic Azad University, Research and Science Branch, Tehran.
} 
on these findings, the moderate intensity aerobic exercises as a useful and medical method for improve the sleep quality among community older adults was recommended.

Key words: Sleep Quality, Aerobic Exercises, Maximum Heart Rate

\section{Introduction}

Sleep is one of the vital aspects for overall health, especially in older adults. Sleep disorders and sleep disturbance are highly problems that reported by $39-75 \%$ of older adults. ${ }^{1-3}$ In the United States, the epidemiologic studies reported that almost half of the older adults have difficulty in sleep onset and maintenance. ${ }^{3,4}$ The most common sleep complaint in older adults is insomnia. ${ }^{5}$ Martin et al. (2004) reported that the direct and indirect costs of insomnia have been estimated at over $\$ 100$ billion per year in the United States. ${ }^{6}$ Studies specific on the older adults indicated that symptoms of insomnia and sleep disturbances are associated with daytime dysfunction, poorer cognitive function and quality of life, depression, activity limitations, fatigue, emotional distress, increased risk of falls and increased incidence of cardiovascular morbidity and mortality. 3,5,7 Furthermore, several studies have showed that older adults spend a smaller percentage of time in rapid eye movement (REM) sleep and a higher percentage of time in light sleep (stages 1 and 2 sleep). ${ }^{8,9}$

One method to improve and increase sleep quality is to take medication, but this method has side-effects (such as daytime residual effects, tolerance, dependence, and rebound insomnia). ${ }^{10}$ Thus, the use of non-medical or non-pharmacological methods appears to be necessary in older adults.

Based on the results of previous studies, one of the useful and without side-effects methods is physical activity. The maintenance of high physical function is one of the key factors for successful aging. ${ }^{11}$ Staying physically and mentally active can not only delay the development of some chronic illnesses and disabilities in older adults, but also improve sleep quality.

In well-controlled laboratory settings, King and colleagues $(1997,2008)$ reported that older adults with moderate sleep complaints can improve and increase sleep quality by moderate-intensity exercise program. ${ }^{13-14}$ Also, previous studies have reported that Tai Chi or yoga activities may improve sleep quality. ${ }^{15-16}$ Although Tai Chi and yoga have been found beneficial in older adults, their complexity makes them difficult for older adults to learn. ${ }^{5}$ On the other hand, laboratory setting cannot provide for all individuals. Therefore, using the aerobic exercise in field setting seem be necessary in older adults. Unfortunately, no study has examined the aerobic exercise effects on sleep quality in field setting, so that all older individuals can use it results and exercise trainings. Thus, the purpose of this study was to examine the effects of low and moderate intensity aerobic exercises on sleep quality in older adults. 


\section{Methods}

Participants: A total of 45 men older adults with ages between 60 to 70 years participated in this research. Subjects were selected through the eligibility components (figure 1) and divided randomly in two experimental groups (aerobic exercise with low and moderate intensity) and one control group.

Eligibility Components: The eligibility components consisted of (a) age between 60 to 70 years, (b) without sleep apnea, (c) not smoking, (d) not engaged particularly in moderate and vigorous physical activity, (e) no taking hypnotic drugs, (f) without any musculoskeletal problems that would prevent participation in aerobic exercises.

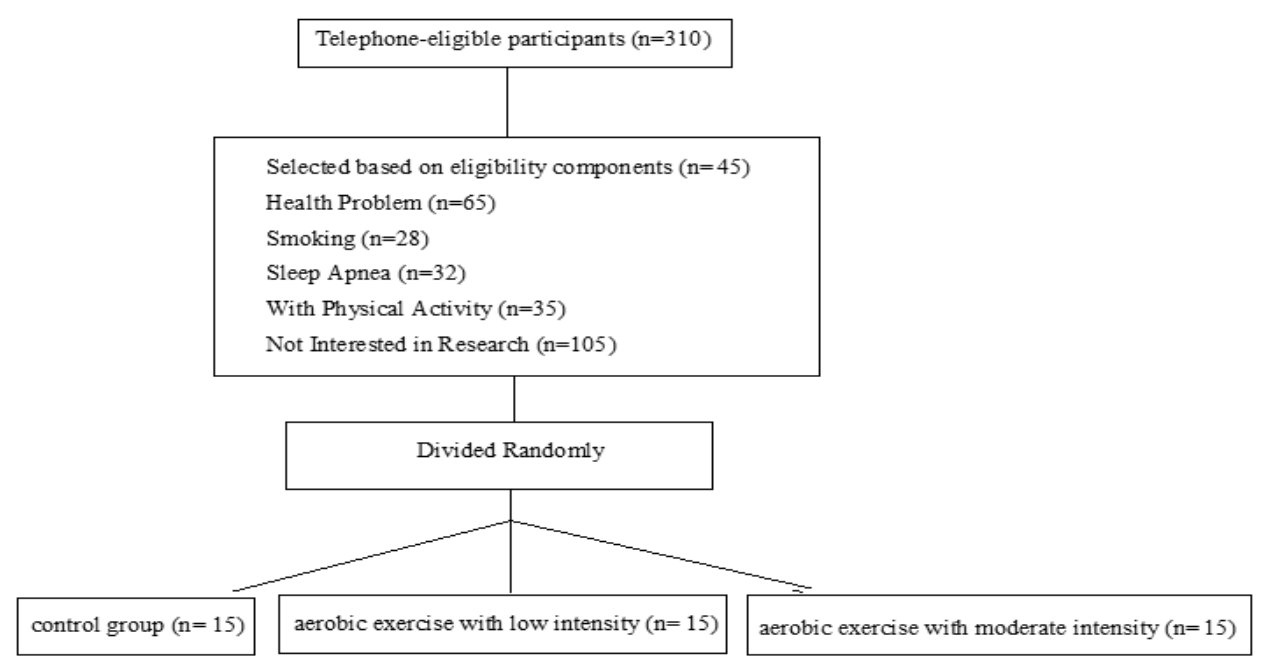

Figure1. Sampling Procedure

Interventions: For safety and risks associated with aerobic exercises in older adults, all of them were evaluated by a doctor to confirm that their physical and mental health. Also, the maximum heart rate (MaxHR) was obtained by subtracting one's age from 220. Furthermore, based on aerobic exercise type (40-50\% MaxHR for low intensity group and 60-70\% MaxHR for moderate intensity group) the target MaxHR was calculated for each subject.

The exercise protocol consisted of 8 weeks aerobic exercises $(2$ sessions in per week) based on Rockport one-mile walking/running test. The aerobic exercise sessions took place in the morning (at approximately $7 \mathrm{am}$ to $10 \mathrm{am}$ ).

Participants in the low intensity (40-50\% MaxHR) and moderate intensity (60$70 \%$ MaxHR) groups were conducted their exercise protocols, and the control group 
continued their daily activities. Moreover, the exercise intensity was evaluated and controlled with uses specific chest belts (Polar X-trainer Plus). The exercise program consisted of warm-up (10 minutes with 20-30\% MaxHR), 35 minutes of low or moderate aerobic exercises and cool-down in 10 minutes, respectively. ${ }^{13,17}$

Also, all sessions by two examiners who have expertise in the field of exercise science and sports physiology had been done. These individual were well expertise in exercise and work with older adults. Furthermore, this study was conducted according to the Ethical Committee of the University of Social Welfare and Rehabilitation Sciences.

Measures: To measure sleep quality, we used the validated Petersburg Sleep Quality Index (PSQI) in pre-test and post-test stages. ${ }^{13-14}$ the PSQI is an 18 -item questionnaire and assesses sleep quality and disturbances in the recent month. It is a Total measure and seven components such as sleep latency, sleep duration, sleep efficacy, sleep disturbance, perceived sleep quality, use of sleep medication and daytime dysfunction. ${ }^{10}$ The score for each components ranges from 0 to 3, whereby 3 reflected the negative extreme on the Likert scale. A score of 5 or greater (indicating a poor sleep) yielded a diagnostic sensitivity of $89.6 \%$ and a specificity of $86.5 \%$, with an internal consistency of 0.83 and test-retest reliability, $\mathrm{r}=0.85$. $^{10,18}$

Data analyze: The collected data have been analyzed with using descriptive (mean and standard deviation) and inferential statistics such as one-way ANOVA and Tokey Post Hoc tests at the $\mathrm{P}<0.05$ significant level with SPSS version 16.

\section{Results}

The descriptive results related to global sleep quality and their components in pre-test and post-test stages are presented in table 1.

Table 1. Global sleep quality and their components scores in pre-test and post-test

\begin{tabular}{|c|c|c|c|}
\hline $0.32 \pm 0.007$ & $0.67 \pm 0.13$ & High intensity Group & \multirow{3}{*}{ Perceived Sleep Quality } \\
\hline $0.53 \pm 0.14$ & $0.73 \pm 0.12$ & Low intensity Group & \\
\hline $0.79 \pm 0.06$ & $0.77 \pm 0.008$ & Control Group & \\
\hline $0.35 \pm 0.10$ & $0.70 \pm 0.18$ & High intensity Group & \multirow{3}{*}{ Sleep Latency } \\
\hline $0.46 \pm 0.16$ & $0.67 \pm 0.15$ & Low intensity Group & \\
\hline $0.74 \pm 0.17$ & $0.68 \pm 0.16$ & Control Group & \\
\hline
\end{tabular}


MODERATE-INTENSITY AEROBIC EXERCISE IMPROVES SLEEP QUALITY IN MEN OLDER $\ldots 13$

\begin{tabular}{|c|c|c|c|}
\hline $0.39 \pm 0.07$ & $0.77 \pm 0.15$ & High intensity Group & \multirow{3}{*}{ Sleep Duration } \\
\hline $0.61 \pm 0.13$ & $0.74 \pm 0.16$ & Low intensity Group & \\
\hline $0.81 \pm 0.14$ & $0.80 \pm 0.15$ & Control Group & \\
\hline $0.37 \pm 0.09$ & $0.71 \pm 0.12$ & High intensity Group & \multirow{3}{*}{ Sleep Efficacy } \\
\hline $0.53 \pm 0.10$ & $0.70 \pm 0.13$ & Low intensity Group & \\
\hline $0.70 \pm 0.12$ & $0.69 \pm 0.11$ & Control Group & \\
\hline $0.33 \pm 0.08$ & $0.73 \pm 0.15$ & High intensity Group & \multirow{3}{*}{ Sleep Disturbance } \\
\hline $0.58 \pm 0.11$ & $0.69 \pm 0.14$ & Low intensity Group & \\
\hline $0.71 \pm 0.13$ & $0.72 \pm 0.16$ & Control Group & \\
\hline $0.36 \pm 0.07$ & $0.81 \pm 0.11$ & High intensity Group & \multirow{3}{*}{ Use of Sleep Medication } \\
\hline $0.54 \pm 0.09$ & $0.79 \pm 0.12$ & Low intensity Group & \\
\hline $0.72 \pm 0.10$ & $0.74 \pm 0.14$ & Control Group & \\
\hline $0.35 \pm 0.12$ & $0.81 \pm 0.22$ & High intensity Group & \multirow{3}{*}{ Daytime Dysfunction } \\
\hline $0.51 \pm 0.10$ & $0.74 \pm 0.24$ & Low intensity Group & \\
\hline $0.72 \pm 0.17$ & $0.73 \pm 0.19$ & Control Group & \\
\hline $2.47 \pm 0.62$ & $5.20 \pm 1.06$ & High intensity Group & \multirow{3}{*}{ Total Sleep Quality } \\
\hline $3.76 \pm 0.83$ & $5.60 \pm 1.07$ & Low intensity Group & \\
\hline $5.19 \pm 0.89$ & $5.12 \pm 0.95$ & Control Group & \\
\hline
\end{tabular}

On the other hand, the pre-test and post-test scores were analyzed with one-way ANOVA test. Results in pre-test stage showed that there are no significant between 3 groups in global sleep quality and their components $(\mathrm{P}>0.05)$. Also, the results in post-test stage showed that there are significant differences between groups in all variables $(\mathrm{P}<0.05)$.

Table 2. One-way ANOVA results in post-test stage

\begin{tabular}{|c|c|c|c|c|c|c|}
\hline \multirow{3}{*}{$0.001 *$} & \multirow{3}{*}{15.750} & 2 & 2.40 & 4.80 & Between Group & \multirow{3}{*}{$\begin{array}{l}\text { Perceived Sleep } \\
\text { Quality }\end{array}$} \\
\hline & & 42 & 0.151 & 6.40 & Within Group & \\
\hline & & 44 & ------ & 11.20 & Total & \\
\hline \multirow{3}{*}{$0.001 *$} & \multirow{3}{*}{9.690} & 2 & 2.42 & 4.82 & Between Group & \multirow{3}{*}{ Sleep Latency } \\
\hline & & 42 & 0.152 & 10.40 & Within Group & \\
\hline & & 44 & ------ & 15.22 & Total & \\
\hline \multirow{3}{*}{$0.001 *$} & \multirow{3}{*}{16.165} & 2 & 2.82 & 5.64 & Between Group & \multirow{3}{*}{ Sleep Duration } \\
\hline & & 42 & 0.175 & 7.33 & Within Group & \\
\hline & & 44 & ------ & 12.97 & Total & \\
\hline
\end{tabular}




\begin{tabular}{|c|c|c|c|c|c|c|}
\hline \multirow{3}{*}{$0.001 *$} & \multirow{3}{*}{11.790} & 2 & 2.02 & 4.04 & Between Group & \multirow{3}{*}{ Sleep Efficacy } \\
\hline & & 42 & 0.171 & 7.20 & Within Group & \\
\hline & & 44 & ------ & 11.24 & Total & \\
\hline \multirow{3}{*}{$0.001 *$} & \multirow{3}{*}{12.710} & 2 & 2.82 & 5.64 & Between Group & \multirow{3}{*}{ Sleep Disturbance } \\
\hline & & 42 & 0.222 & 9.33 & Within Group & \\
\hline & & 44 & ----- & 14.91 & Total & \\
\hline \multirow{3}{*}{$0.001 *$} & \multirow{3}{*}{15.750} & 2 & 2.41 & 4.81 & Between Group & \multirow{3}{*}{$\begin{array}{l}\text { Use of Sleep } \\
\text { Medication }\end{array}$} \\
\hline & & 42 & 0.153 & 6.45 & Within Group & \\
\hline & & 44 & ----- & 11.26 & Total & \\
\hline \multirow{3}{*}{$0.001 *$} & \multirow{3}{*}{10.450} & 2 & 2.83 & 5.66 & Between Group & \multirow{3}{*}{ Daytime Dysfunction } \\
\hline & & 42 & 0.270 & 11.33 & Within Group & \\
\hline & & 44 & ------ & 16.99 & Total & \\
\hline \multirow{3}{*}{$0.001 *$} & \multirow{3}{*}{110.076} & 2 & 123.356 & 246.711 & Between Group & \multirow{3}{*}{ Total Sleep Quality } \\
\hline & & 42 & 1.122 & 47.067 & Within Group & \\
\hline & & 44 & ------ & 293.778 & Total & \\
\hline
\end{tabular}

*Significant level at the $\mathbf{P}<0.05$

Also, the Tukey Post Hoc showed that the moderate intensity group scores in sleep quality and its components were better than other groups $(\mathrm{P}<0.05)$. Finally, the low intensity group scores were better than control group $(\mathrm{P}<0.05)$. For more details see tables 1 and 2.

\section{Discussion}

Sleep disturbance is one of the important problems that are caused by age related changes in human. With increasing age, inappropriate changes are created in sleep structure and sleep cycle. So that, studies have shown that poor sleep quality in older adults is directly associated with physical and mental impairments. ${ }^{13,19}$ Thus the purpose of this study was to investigate the effect of low and moderate intensity aerobic exercises on sleep quality and its components in men older adults.

Our results from this study indicate that eight-week moderate-intensity aerobic exercise was effective on improving sleep quality and all its components in men older adults. These results highlight the potential of structured field aerobic exercise programs to improve these variables. Our results are congruent with well-controlled laboratory researches. ${ }^{11,13}$ Accordingly, King and colleagues (2008) in their study found that the moderate-intensity endurance exercise may have modest positive effects on several dimensions of sleep quality aspects. Researchers reported that the 12-month exercise with moderate-intensity shift observed from Stage 1 to Stage 2 sleep and the reduced number of awakenings observed during this sleep phase. ${ }^{13}$ Furthermore, Reid et al. (2010) reported that the 6-week program of moderate aerobic exercise plus sleep hygiene education 
is effective in improving self reported sleep quality, mood and quality of life in older adults with insomnia. In this study, the increase in sleep duration by 1.25 hours was higher than what has been reported for other non-pharmacological interventions for insomnia. 11

The mechanisms underlying these results could be explained by the thermoregulatory and energy conservation theories. ${ }^{13,19}$

Murphy \& Campbell (1997) argued that that sleep onset is the evening decline in body temperature, which is primarily mediated by increased peripheral skin blood flow. ${ }^{20}$ Sleep onset is associated with peripheral heat dissipation through vasodilation and increased sweating, together with a reduction in metabolic rate and core body temperature during sleep. 11,21 Furthermore, body temperature is regulated at a lower level during non-REM sleep than during wakefulness while thermoregulation is inhibited during REM sleep. ${ }^{21}$ Also, in this mechanism, the anterior hypothalamus region plays a vital role in sleep and temperature regulation, ${ }^{22}$ so that body temperature elevation before bed-time can activate both heat-loss and the associated sleep mechanisms. 11,22

On the other hand, there are two types of the energy conservation theory. One type is that sleep is for the reduction of energy expenditure below the level attainable by rest alone ${ }^{11}$, the second type suggests that the sleep sets a limit on metabolic expenditure to the extent necessary to balance a species' energy budget. ${ }^{11,23}$ some evidence related to the energy conservation function for sleep is that energy expenditure is only $10-15$ percents less than that associated with quiet wakefulness.

Taken together, we conclude that the field moderate-intensity aerobic exercise has a positive effect on sleep quality and its components. Thus, the field moderateintensity aerobic exercise as a useful and therapeutic method is recommended for older adults.

\section{References}

1. Vaz Fragoso, A., \& Gill, M. Sleep complaints in community-living older persons: A multi-factorial geriatric syndrome. Journal of the American Geriatric Society, 2007, 55, 1853-1866.

2. Institute of Medicine of the National Academy of Sciences. Sleep disorders and sleep deprivation: An unmet public health problem. Washington, DC: National Academies Press. 2006.

3. Freburger, J., Callahan, L., Shreffler, H., \& Mielenz, T. The effects of a physical activity program on sleep and health-related quality of life in older persons with arthritis. Journal of Applied Gerontology, 2010, 29(4) 395-414.

4. Foley, J., Monjan, A., Brown, L., Simonsick, M., Wallace, B., \& Blazer, D. Sleep complaints among elderly persons: An epidemiologic study of three communities. Sleep, $1995,18,425-432$. 
5. Chen, M., Liu, H., Huang, H., \& Chiou, A.F. The effect of a simple traditional exercise program (Baduanjin exercise) on sleep quality of older adults: A randomized controlled trial. International Journal of Nursing Studies, 2012, 49, 265-273.

6. Martin, S., Aikens, J., \& Chervin, D. Toward cost-effectiveness analysis in the diagnosis and treatment of insomnia. Sleep Medicine Reviews, 2004, 8(1), 63-72.

7. Blackwell, T., Yaffe, K., Ancoli-Israel, S., Schneider, L., Cauley, A., Hillier, A., et al. Poor sleep is associated with impaired cognitive function in older women: The study of osteoporotic fractures. Journal of Gerontology: Biological Sciences, 2006, 61A, 405-410.

8. Neikrug B., Ancoli-Israel S. Sleep disorders in the older adult - a mini-review. Gerontology 2010; 56:181-9.

9. Santos, R., Viana, V., Boscolo, A., Marques, G., Santana, G., Lira, S., Tufik, S. \& de Mello, T. Moderate exercise training modulates cytokine profile and sleep in elderly people. Cytokine 2012, 60, 731-735.

10. Lai, H. Music improves sleep quality in older adults. Journal of Advanced Nursing, 2006, 49 (3), 234-244.

11. Reid J., Baron, K., Lu, B., Naylor, E., Wolfe, L., \& Zee, Ph. Aerobic exercise improves self-reported sleep and quality of life in older adults with insomnia. Sleep Medicine, 2010, 934-940.

12. Kramer A.F., Hahn S., Cohen N.J., Banich M.T., McAuley E., Harrison C.R., et al. Ageing, fitness and neuro-cognitive function. Nature 1999; 400: 418-419.

13. King, C. Pruitt, L., Woo, S., Castro, C., Ahn, D., Vitiello, M., Woodward, S., \& Bliwise, D. Effects of Moderate-Intensity Exercise on Polysomnographic and Subjective Sleep Quality in Older Adults With Mild to Moderate Sleep Complaints. Journal of Gerontology: Medical Sciences, 2008, 63(9), 997-1004.

14. King C., Oman F., Brassington S., Bliwise L., \& Haskell L. Moderate-intensity exercise and self-rated quality of sleep in older adults. A randomized controlled trial. JAMA, 1997; 277(1): 32-37.

15. Chen, M., Chen, H., Chao, C., Hung, M., Lin, S.,\& Li, H., Sleep quality, depression state, and health status of older adults after silver yoga exercises: cluster randomized trial. International Journal of Nursing Studies. 2009, 46(2), 154-163

16. Irwin, R., Olmstead, R., \& Motivala, J. Improving sleep quality in older adults with moderate sleep complaints: a randomized controlled trial of Tai Chi Chih. Sleep, 2008. 31(7), 1001-1008.

17. Roveda, E., Sciolla, C., Montaruli, A., Calogiuri, G., Angeli, A., Carandente, F., Effects of endurance and strength acute exercise on night sleep quality. International Sport Medicine Journal, Vol.12 No.3, 2011, pp. 113-124.

18. Buysse, J., Reynolds, F., Monk, H., Berman, R.,\& Kupfer, J. The Petersburg Sleep Quality Index: a new instrument for psychiatric practice and research. Psychiatry Research, 1989, 28(2), 193-213.

19. Driver, H., \& Taylor, S. Exercise and sleep. Sleep Medicine Reviews, 2000, 4(4), 387-402.

20. Murphy, J., \& Campbell, S. Nighttime drop in body temperature: a physiological trigger for sleep onset? Sleep, 1997; 20: 505-511. 
21. Glotzbach, S., \& Heller, C. Temperature Regulation. In: Kryger MH, Roth T, Dement WC(eds). Principles and Practice of Sleep Medicine. Philadelphia: W.B. Saunders Co. 1994, 260-275.

22. McGinty, D., \& Szymusiak, R. Keeping cool, a hypothesis about the mechanisms and functions of slow-wave sleep. Trends Neuroscience, 1990; 13: 480-487.

23. Zepelin, H. Mammalian sleep. In: Kryger MH, Roth T, Dement WC(eds). Principles and Practice of Sleep Medicine. Philadelphia: W.B. Saunders Co., 1994, 69-80. 